\title{
Next generation antidepressants
}

Scott J. Russo ${ }^{a, b, 1}$ and Dennis S. Charney ${ }^{a, b, c, 1}$

${ }^{\mathrm{a}}$ Fishberg Department of Neuroscience and Friedman Brain Institute, ${ }^{\mathrm{b}}$ Graduate School of Biomedical Sciences, and 'Department of Psychiatry, Icahn School of Medicine at Mount Sinai, New York, NY 10029

Over the past $50 \mathrm{y}$, there have been few mechanistically distinct drugs for the treatment of major depressive disorders, despite

\section{the fact that nearly two-thirds of patients do not achieve full remission of symptoms on currently available antidepressants (1)}

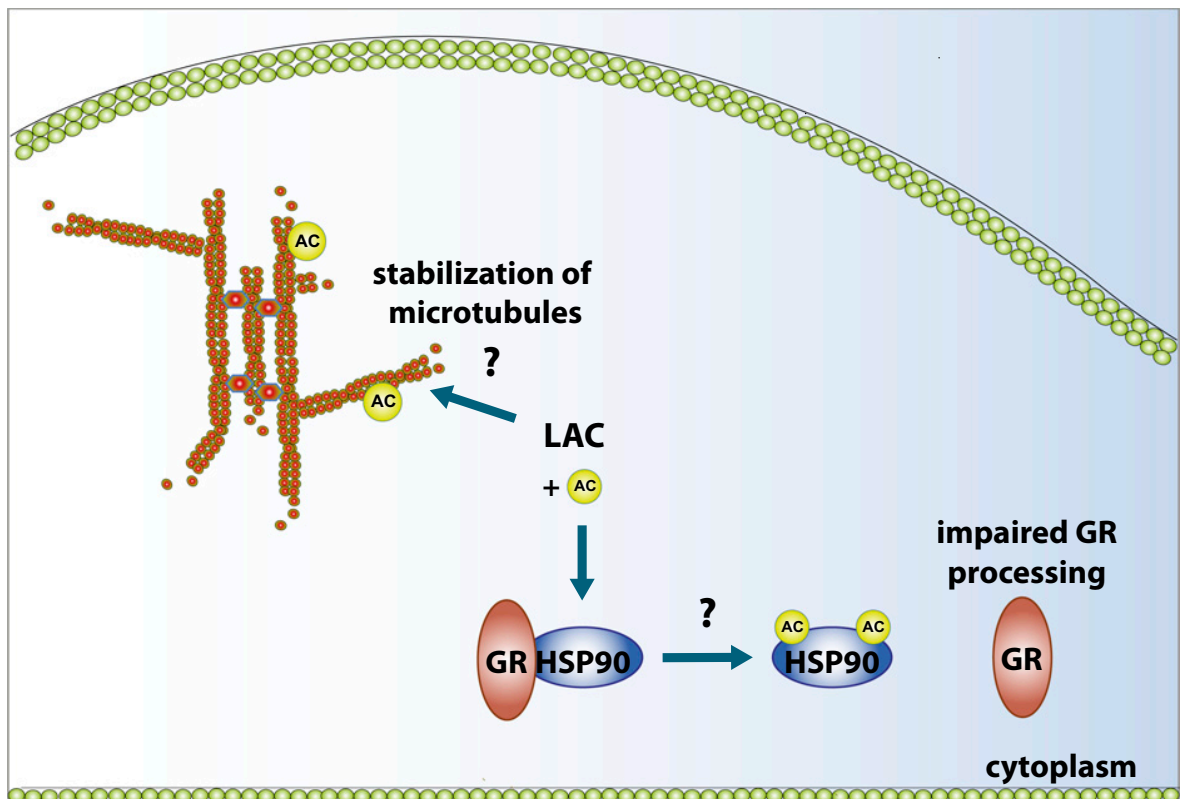

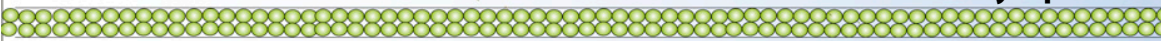

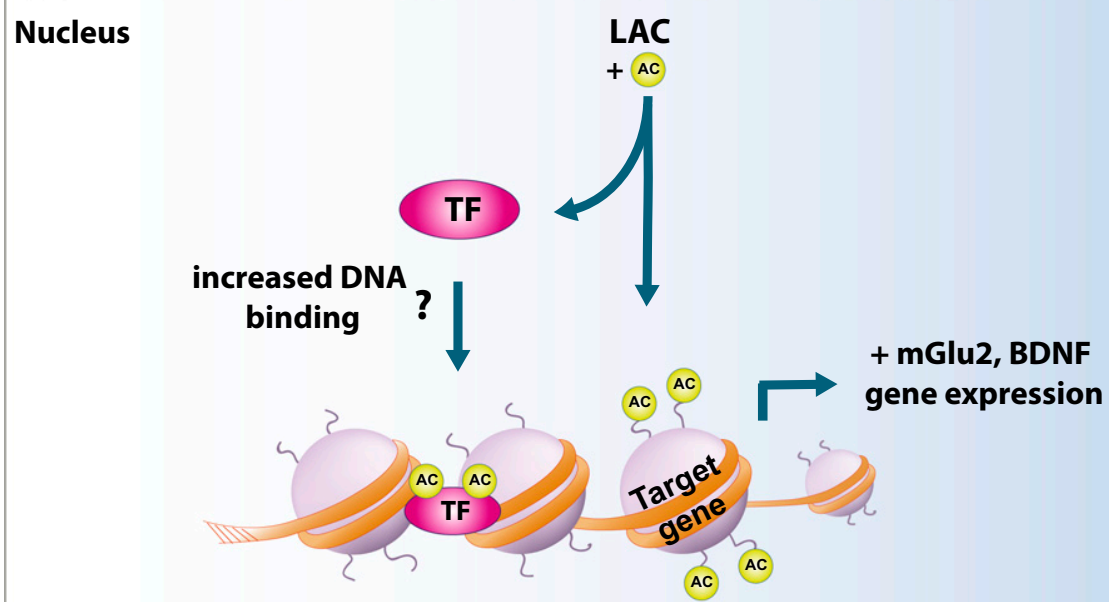

Fig. 1. Schematic describing potential cellular mechanisms for rapid antidepressant properties of L-acetylcarnitine. LAC may act within the nucleus to promote gene expression of mGlu2 receptor and BDNF by acetylation (AC) of transcription factors (TF) that promote DNA binding or of histones proteins surrounding DNA, leading to a permissive transcriptional state. LAC may act outside the nucleus on chaperone proteins, such as HSP90, to impair glucocorticoid receptor (GR) processing and limit the cellular effects of stress. LAC may acetylate microtubule proteins in dendrites to control stability of the neuronal cytoskeleton and prevent stress-induced loss of dendrites.
In addition, even when adequate remission is achieved, patients require $2-4 \mathrm{wk}$ of treatment before any significant effects, increasing the risk for complications, such as suicide (2). This delay in effectiveness has resulted in a major push to identify and develop novel therapeutics with more rapid effects. The recent identification of ketamine as a rapid antidepressant effective in treatment-resistant patients has been groundbreaking $(3,4)$. Nasca et al. (5) describe in PNAS a unique potential rapidly acting antidepressant, L-acetylcarnitine (LAC), which is a dietary supplement that acts by acetylating protein targets to control their function. LAC is reported to be well tolerated and can readily cross the blood-brain barrier (6). A recent study suggests it has promise in the treatment of Parkinson disease because of its neroprotective properties (7). Strikingly, LAC exhibits antidepressant efficacy within 2-3 d following intraperitoneal administration in rodents, compared with 2-3 wk with a standard antidepressant treatment, such as chlorimipramine. Although LAC is relatively nonspecific and can target many biological pathways, it is suggested by Nasca et al. (5) to promote rapid antidepressant responses by acetylation of histone proteins that control the transcription of $B D N F$ and metabotropic glutamate $2(m G l u 2)$ receptors in the hippocampus (Hipp) and prefrontal cortex (PFC) (Fig. 1). One of the more impressive aspects of this article is that Nasca et al. (5) verify rapid antidepressant efficacy of LAC in both a genetic rat model of susceptibility [Flinders Sensitive Line (FSL)] and following chronic stress exposure, factors that are thought to be the primary cause of depression in humans. Although there is clearly far more work necessary to understand the mechanisms of antidepressant action of LAC in rodents, and the dose and relative safety profile for

Author contributions: S.J.R. and D.S.C. wrote the paper.

Conflict of interest statement: D.S.C. and Icahn School of Medicine at Mount Sinai have been named on a use patent application of Ketamine for the treatment of depression. If Ketamine was shown to be effective in the treatment of depression and received approval from the Food and Drug Administration (FDA) for this indication, D.S.C. and Icahn School of Medicine at Mount Sinai could benefit financially.

See companion article on page 4804

${ }^{1}$ To whom correspondence may be addressed. E-mail: scott.russo@ mssm.edu or dennis.charney@mssm.edu. 
depression treatment in humans, these exciting results are a first step toward that goal.

There is a growing literature suggesting that chronic stress reprograms gene expression in brain regions, such as the Hipp and nucleus accumbens (NAc), and that normalization of the transcriptional machinery through posttranslational modifications of histones can correct some of the aberrant gene transcription and promote antidepressant responses (8). Histone deacetylase (HDAC) inhibitors given intraperitoneally $(9,10)$, as well as directly into the Hipp $(11)$, amygdala (11), and NAc $(12,13)$, promote antidepressant behavioral responses following stress. In the NAc, HDAC inhibitors have been shown to normalize gene-expression profiles similar to chronic imipramine-treated and control unstressed mice. Work described by Nasca et al. (5) suggests similar mechanisms of action for LAC, whereby increased histone acetylation and normalization of BDNF or mGlu2 in the Hipp and PFC promotes resilience following chronic unpredictable stress, or in the FSL rats. Interestingly, the authors show that normalization of mGlu2 receptor expression through an epigenetic mechanism is functionally related to antidepressant responses (5); HDAC inhibitors normalize mGlu2 expression in FSL rats and LAC has limited antidepressant efficacy in $m$ Glu 2 knockout mice. These findings are very interesting; however, there are some important questions to consider. For example, previous work has shown that increased BDNF in the hippocampus is antidepressant (14), but increased BDNF in the mesolimbic dopamine system is prodepressant (15). Does peripheral administration of LAC also increase BDNF transcription in the mesolimbic dopamine system through a similar mechanism, and does this increase the possibility of unwanted side effects or limited efficacy? Furthermore, there are likely many other epigenetic targets across multiple brain areas contributing to the antidepressant behavioral effects of LAC that warrant further investigation.

Nasca et al. (5) point out the possibility that LAC may acetylate transcription factors, such as NFKB, to regulate mGlu2 transcription (Fig. 1). Their results suggest that increased acetylation of p65 by LAC is associated with its antidepressant efficacy and that pretreatment with the anti-inflammatory sodium salicylate, which nonselectively inhibits NFKB, blocks the effects of LAC on mGlu2 gene transcription, and partially blocks antidepressant behavioral responses. These findings are somewhat contrary to previous work, which shows that chronic stress increases NFKB-dependent transcription or its upstream kinase, IKK, in the Hipp (16) and NAc (17), respectively. In the Hipp, increased NFkB-dependent transcription promotes stress-impaired neurogenesis, but local administration of a selective NFkB inhibitor prevents stress-impaired neurogenesis and shows antidepressant efficacy on noveltyinduced hypophagia and sucrose consumption, which are classic depression and anxiety tests (16). In the NAc, IKK inhibition reverses social defeat stress-induced social-avoidance behavior and synaptic plasticity (17). Thus, although the current findings are intriguing, they raise a number of important questions. First, sodium salicylate acts on many more targets other than NFKB. Is sodium salicylate acting through these other targets to control $m$ Glu2 transcription and antidepressant behavioral responses? The use of more specific NFKB inhibitors or genetic knockouts will be important to determine this Next, although Nasca et al. (5) mention that the $m G l u 2$ promoter contains a number of NFкB binding sites, it's unclear whether LAC promotes acetyl-p65 binding to the $m$ Glu2 promoter in vivo and whether this increases $m G l u 2$ transcription.

Finally, it is important to note that LAC is a nonspecific acetylating agent and it is unclear from the present work whether the antidepressant actions are solely a result of acetylation of nuclear proteins, such as transcription factors and histones, or whether it is in part a result of acetylation of other proteins outside the nucleus (Fig. 1). For example, acetylation of microtubules can influence the stability of the neuronal cytoskeleton (18). Chronic stress and genetic

1 Preskorn SH (2012) Ketamine: The hopes and the hurdles. Biol Psychiatry 72(7):522-523.

2 Rush AJ, et al. (2009) STAR*D: Revising conventional wisdom. CNS Drugs 23(8):627-647

3 Berman RM, et al. (2000) Antidepressant effects of ketamine in depressed patients. Biol Psychiatry 47(4):351-354

4 Murrough JW, et al. (2012) Rapid and longer-term antidepressant effects of repeated ketamine infusions in treatment-resistant major depression. Biol Psychiatry, 10.1016/j.biopsych.2012.06.022 5 Nasca C, et al. (2013) L-acetylcarnitine causes rapid antidepressan effects through the epigenetic induction of mGlu2 receptors. Proc Natl Acad Sci USA 110:4804-4809.

6 Barhwal K, et al. (2009) Acetyl---carnitine (ALCAR) prevents hypobaric hypoxia-induced spatial memory impairment through extracellular related kinase-mediated nuclear factor erythroid 2-related factor 2 phosphorylation. Neuroscience 161(2):501-514. 7 Beal MF (2003) Bioenergetic approaches for neuroprotection in Parkinson's disease. Ann Neurol 53(Suppl 3):S39-S47; discussion S47-S48

8 Tsankova N, Renthal W, Kumar A, Nestler EJ (2007) Epigenetic regulation in psychiatric disorders. Nat Rev Neurosci 8(5):355-367. 9 Schroeder FA, Lin CL, Crusio WE, Akbarian S (2007) Antidepressant-like effects of the histone deacetylase inhibitor sodium butyrate, in the mouse. Biol Psychiatry 62(1):55-64. 10 Tsankova NM, et al. (2006) Sustained hippocampal chromatin regulation in a mouse model of depression and antidepressant action. Nat Neurosci 9(4):519-525.

11 Covington HE, 3rd, Vialou VF, LaPlant Q, Ohnishi YN Nestler EJ (2011) Hippocampal-dependent antidepressant-like models of depression are well-established regulators of the neuronal cytoskeleton, and in the PFC and Hipp, depressive phenotypes have generally been associated with a destabilization of the cytoskeleton and a loss of excitatory glutamatergic synapses (19). Thus, it is tempting to speculate that LAC may affect stability of the cytoskeleton and glutamatergic synapses during times of stress or in genetically predisposed individuals. The possibility that acetylation may affect posttranscriptional processes in stress disorders is not completely speculative. Recent work has identified a role for the histone deactylase, HDAC6, outside the nucleus through its deactylating actions on heat-shock protein 90 (HSP90), a chaperone protein important for protein processing (20). Standard antidepressants down-regulate HDAC levels, resulting in hyperacetylation of HSP90 and subsequent impairment of glucocorticoid receptor processing, which blunts the impact of stress on serotonergic neurons in the dorsal raphe nucleus. Although these two examples highlight the diverse roles of acetylation on the function of neuronal systems, future work to determine the effects of LAC on a host of nonnuclear targets needs further investigation.

Work by Nasca et al. (5) represents the type of basic science research that can help guide the development of novel, more rapidly acting treatments for depression. The identification of rapidly acting antidepressants, like ketamine, has resulted in a much needed major paradigm shift in the discovery process that promises to lead to a new generation of antidepressant therapies.

activity of histone deacetylase inhibition. Neurosci Lett 493(3): 122-126.

12 Covington HE, 3rd, et al. (2009) Antidepressant actions of histone deacetylase inhibitors. J Neurosci 29(37):11451-11460. 13 Golden SA, et al. (2013) Epigenetic regulation of RAC1 induces synpatic remodeling in stress disorders and depression. Nat Med, 10.1038/nm.3090.

14 Shirayama Y, Chen AC, Nakagawa S, Russell DS, Duman RS (2002) Brain-derived neurotrophic factor produces antidepressant effects in behavioral models of depression. I Neurosci 22(8): 3251-3261

15 Berton O, et al. (2006) Essential role of BDNF in the mesolimbic dopamine pathway in social defeat stress. Science 311(5762): 864-868

16 Koo JW, Russo SJ, Ferguson D, Nestler EJ, Duman RS (2010) Nuclear factor-kappaB is a critical mediator of stress-impaired neurogenesis and depressive behavior. Proc Natl Acad Sci USA 107(6):2669-2674

17 Christoffel DJ, et al. (2011) IkB kinase regulates social defeat stress-induced synaptic and behavioral plasticity. J Neurosci 31(1): 314-321.

18 Sudo H, Baas PW (2010) Acetylation of microtubules influences their sensitivity to severing by katanin in neurons and fibroblasts. $J$ Neurosci 30(21):7215-7226.

19 Christoffel DJ, Golden SA, Russo SJ (2011) Structural and synaptic plasticity in stress-related disorders. Rev Neurosci 22(5): 535-549.

20 Espallergues J, et al. (2012) HDAC6 regulates glucocorticoid receptor signaling in serotonin pathways with critical impact on stress resilience. J Neurosci 32(13):4400-4416. 